\title{
REVIEW ON ANASTOMOTIC LEAK RATE AFTER ICG ANGIOGRAPHY DURING MINIMALLY INVASIVE COLORECTAL SURGERY
}

\section{Tsanko I. Yotsov', Martin P. Karamanliev ${ }^{1}$, Svilen I. Maslyankov', Dobromir D. Dimitrov ${ }^{1}$}

\author{
Angel Kanchev University of Ruse \\ ${ }^{1}$ Medical University - Pleven, \\ Dr G. Stranski University Hospital - \\ Pleven \\ ${ }^{2}$ Department of Surgical Oncology, \\ Medical University - Sofia
}

\author{
Corresponding Author: \\ Tsanko I. Yotsov \\ Department of Nursing Care, \\ Angel Kanchev University of Ruse \\ 20 Major Atanas Uzunov Str. \\ Ruse, 7000 \\ e-mail: tsankoyotsov@gmail.com
}

Received: August 23, 2021

Revision received: September 13, 2021

Accepted: November 25. 2021

\section{Summary}

Colorectal cancer is the $3^{\text {rd }}$ most common type of cancer worldwide. The most devastating complication after colorectal surgery remains the anastomotic leak (AL). Many techniques have been developed to reduce its rate. One such new method is perfusion angiography using indocyanine green (ICG). A literary search in PUBMED on 1.03.2021 for full-text English articles published between 2014 and 2021 was performed. ICG, colorectal cancer, and angiography were the keywords we used. The review was performed following the recommendations of the Preferred Reporting Items for Systematic Reviews and Meta-Analyses guidelines. The literature search yielded 27 results when searching the database of PUBMED with the above keywords. Twentyone out of 27 identified articles were included. Six were excluded from the analysis - four case reports, one review on the evolution of treating gastrointestinal cancers, and one containing no information on AL rate with ICG. One included article was RCT, sixteen were cohort studies, and four were meta-analyses or reviews. All articles reported a reduction in the anastomotic leak rate. However, the reduction was significant only in nine of them. Anastomotic leak is a severe complication and a subject of extensive research. Perfusion angiography with ICG is a step towards predicting and preventing AL, although it does not guarantee success in all cases.

Keywords: anastomotic leak, ICG angiography, colorectal cancer, minimally invasive surgery

\section{Introduction}

Colorectal cancer is the third most common type of cancer worldwide, representing $10.2 \%$ of the newly diagnosed cancer cases and the second cause of cancer-related death [1]. Colorectal cancer surgery is still a widely researched area and a topic for many discussions. Some innovations in the field have been introduced to tackle difficulties during surgery or complications in the postoperative period. The most devastating complication after colorectal surgery remains the anastomosis leak (AL). Besides higher morbidity and mortality, AL is also associated with higher costs and economic impact. According to a report on data from the Premier Perspective Data database, AL patients' treatment cost is nearly three times higher than the cost of no leak patients [2,3]. There are many risk factors for $\mathrm{AL}$, such as male sex, tobacco 
use, immunosuppression, obesity, preoperative radiotherapy, diabetes, and ischemia of the bowel in the anastomosis area [4,5]. Adequate bowel perfusion is mandatory for proper healing of the tissue after a colon or rectal resection. The blood supply of the segments determined for anastomosis can be objectively evaluated by performing immunofluorescent angiography with indocyanine green (ICG). The popularity of this method is growing, and the benefit of its use is increasingly recognized. The technique is easy to perform, and the results are easy to interpret.

\begin{abstract}
Aim
This paper aimed to systematically review the available data regarding the impact of perfusion angiography with ICG on the anastomotic leak rate after minimally invasive surgery for colorectal cancer.
\end{abstract}

\section{Materials and Methods}

The review was performed following the recommendations of the Preferred Reporting Items for Systematic Reviews and MetaAnalyses (PRISMA) standard guidelines [6check, and reanalyze individual-level data from all studies addressing a particular research question and are therefore considered a gold standard approach to evidence synthesis. They are likely to be used with increasing frequency as current initiatives to share clinical trial data gain momentum and may be particularly important in reviewing controversial therapeutic areas. OBJECTIVE: to develop PRISMA-IPD as a stand-alone extension to the PRISMA (Preferred Reporting Items for Systematic Reviews and Meta-Analyses].

\section{Protocol}

A literary search in PUBMED on 1.03.2021 for full-text English articles published from 2014 to 2021 was performed. The keywords used for the search were ICG, colorectal cancer, and angiography.

\section{Eligibility criteria}

The inclusion criteria for the study were full-text papers reporting cohorts including colorectal patients aged $>18$, performance of minimally invasive procedure, use of ICG angiography for assessing perfusion, and AL rate. Articles reporting only individual cases were excluded. All the researchers independently reviewed the data.

\section{Data extraction}

We extracted data on the type of study from the selected papers, the number of patients $(n=)$ included, percentage of cancer patients, ICG vs. non-ICG AL rate, and statistically significant difference.

\section{Evaluation of study quality and the risk of bias assessment}

The quality of the included articles was assessed according to the 2011 Oxford Centre for Evidence-Based Medicine (OCEBM) levels of evidence [7]. The bias was assessed by the ROBINS-I tool for non-randomized studies of interventions [8].

\section{Endpoints}

The primary endpoint of this review was to assess whether using ICG angiography reduces the $\mathrm{AL}$ rate after minimally invasive surgery in colorectal cancer patients. The secondary endpoint was to investigate whether this reduction was statistically significant in the papers reviewed.

\section{Results}

\section{Literature search}

The literature search on 1.03.2021 in PUBMED from 2014 to 2021 found 27 results when searching the database with ICG, colorectal cancer, and angiography as keywords. From the 27 identified articles, 21 were included. Six communications were excluded from the analysis - four case reports [9-12] GmbH \& Co. $\mathrm{KG}$, Tuttlingen, Germany, one review on the evolution of treating gastrointestinal cancers [13], and one containing no data about AL rate with ICG [14]during colorectal resections. Methods: FLER analysis and visualization was performed in 22 patients (diverticulitis $\mathrm{n}=17$; colorectal cancer $n=5$. (Figure 1 )

The summary of the extracted data from the papers included is presented in Table 1.

Most of the studies were cohort studies that either followed up patients prospectively or gathered information retrospectively, and 


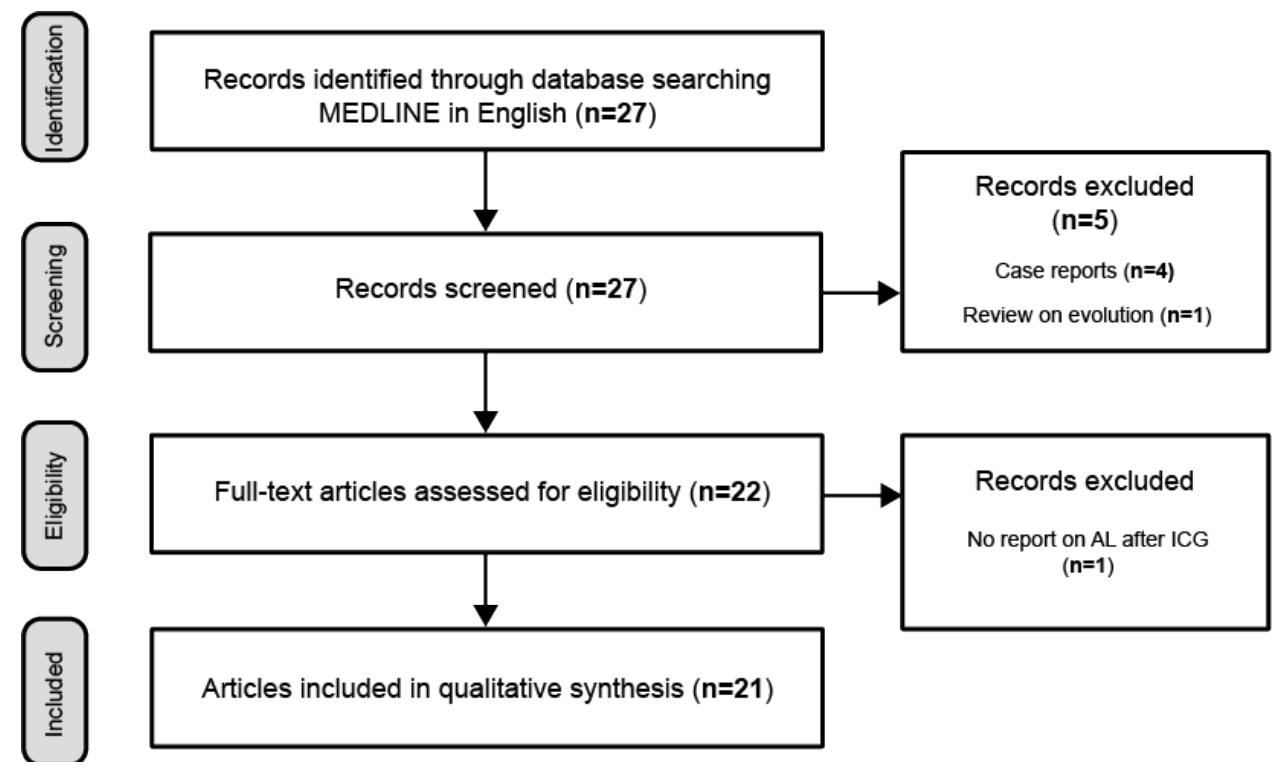

Figure 1. Articles selection flowchart

there was only one randomized controlled trial. Such an approach reduces to a certain extent the quality of the data reported. The number of included patients varied. However, most studies reported cohorts with $100 \%$ cancer patients. Only one study reported data with less than 50\% cancer patients in the cohort [29]tension free, accurate tissue apposition, and minimal local spillage. Traditionally, perfusion is measured by assessing palpable pulses in the mesentery, active bleeding at cut edges, and lack of tissue discoloration. However, subjective methods lack predictive accuracy for an anastomotic leak. We used intraoperative indocyanine green (ICG. The data from Table 1 shows that most of the studies reported a reduction in AL rate when comparing ICG and non-ICG groups with a varying degree of $0 \%$ to $9 \%$. Two of the four reviewed meta-analyses reported pooled data from the studies included. They also reported statistical significance in the reduction of $\mathrm{AL}$ rates when the ICG perfusion test was performed. When reviewed, the two meta-analyses that did not report pooled data still showed a reduction in AL rates in the individual studies analyzed. In total, eight of the studies showed that using ICG angiography provided a statistically significant reduction in AL rate, four reported no statistical significance, and the rest of the studies did not report statistical significance at all. The authors of one of the studies, reporting no significance, attributed the result to the limited number of patients included [26)increasing in lower anastomoses. Many etiological factors have been evaluated and most of these are related to bowel perfusion. Indocyanine green-enhanced fluorangiography (ICGf. Four articles even reported a $0 \%$ leak rate $[15,19,25,31]$ no studies have been published in the literature evaluating the impact of the ICG-FA in the ERAS protocol for the patients suitable for colorectal surgery. The aim of our study was to assess whether the systematic evaluation of intestinal perfusion by ICG-FA could improve patients outcomes when managed with ERAS perioperative protocol, thus reducing surgical complication rate. This is a retrospective case-control study. From March 2014 to April 2017, 182 patients underwent laparoscopic colorectal surgery for benign and malignant diseases. All the patients were enrolled in ERAS protocol. Two groups were created: Group A comprehended 107 patients managed within the ERAS pathway only and Group B comprehended 75 patients managed as well as with ERAS pathway plus the intraoperative assessment of intestinal perfusion with ICGFA. Two board-certified laparoscopic colorectal surgeons jointly performed all procedures. Six (5.6\%. In a study of 488 colorectal cancer patients [20], the reported AL rates were $1.8 \%$ vs. 5.3\% when comparing ICG with non-ICG patients, which difference was statistically insignificant.

Sub-analysis was performed, and the rectal cancer patients were separated from the colon 
Table 1. Extracted data from articles

\begin{tabular}{|c|c|c|c|c|c|c|}
\hline № & Author & Type of study & $\mathbf{n}=$ & $\% \mathrm{CP}$ & ICG vs. non-ICG & Significance \\
\hline 1. & Brescia et al. [15] & Cohort study & 182 & 89 & $0 \%$ vs. $5.6 \%$ & Yes \\
\hline 2. & De Nardi et al. [16] & RCT & 252 & 75.25 & $5 \%$ vs. $9 \%$ & No \\
\hline 3. & Hasegawa et al. [17] & $\begin{array}{l}\text { Propensity } \\
\text { score-matched }\end{array}$ & $\begin{array}{l}852[420 \\
\text { after PSM) }\end{array}$ & 100 & $2.8 \%$ vs. $13.6 \%$ & Yes \\
\hline 4. & Son et al. [18] & Cohort study & 86 & 100 & $\begin{array}{l}7 \% \text { (quantitative, not } \\
\text { comparative) }\end{array}$ & Not reported \\
\hline 5. & Boni et al.[19] & Case-matched & 42 & 100 & $0 \%$ vs. $5 \%$ & No \\
\hline 6. & Ishii et al.[20] & Cohort study & 488 & 100 & $\begin{array}{l}1,8 \% \text { vs. } 5.3 \% \text { (all) } \\
3.5 \% \text { vs. } 10.5 \% \text { (rectal) }\end{array}$ & $\begin{array}{l}\text { Yes, for rectal } \\
\text { cancer }\end{array}$ \\
\hline 7. & Zhang et al. [21] & Meta-analysis & 6312 & $\begin{array}{l}\text { Not } \\
\text { reported }\end{array}$ & $3.22 \%$ vs. $9.17 \%$ & Yes \\
\hline 8. & Santi et al. [22] & Cohort study & 38 & 100 & $2.63 \%$ vs. - & Not reported \\
\hline 9. & Liu et al. [23] & $\begin{array}{l}\text { Systematic } \\
\text { review+ Meta- } \\
\text { analysis }\end{array}$ & 4037 & 100 & $3.8 \%$ vs. $7.8 \%$ & Yes \\
\hline 10 . & Lin et al. [24] & Meta-analysis & 3137 & 100 & No pooled data & Yes \\
\hline 11. & Boni et al. [25] & Cohort study & 107 & 90 & $0 \%$ & Not reported \\
\hline 12. & Bonadio et al. [26] & Cohort study & 66 & 100 & $6 \%$ vs. $21 \%$ & No \\
\hline 13. & Benčurik et al. [27] & Cohort study & 100 & 100 & $9 \%$ vs. $19 \%$ & Yes \\
\hline 14. & Carus et al. [28] & Cohort study & 378 & 100 & $3.7 \%$ vs $7.1 \%$ & Not reported \\
\hline 15. & Protyniak et al. [29] & Case review & 77 & 44 & $2.6 \%$ & Not reported \\
\hline 16. & Li et al. [30] & Meta-analysis & 2593 & 100 & No pooled data & Yes \\
\hline 17. & Ris et al. [31] & Cohort study & 30 & 83.3 & $0 \%$ & Not reported \\
\hline 18. & Gröne et al. [32] & Cohort study & 18 & 100 & $6 \%$ & Not reported \\
\hline 19. & Wada et al. [33] & Cohort study & 149 & 100 & $\begin{array}{l}10.4 \% \text { vs } 6.9 \% \text { before PSM } \\
8.8 \% \text { vs } 14,7 \% \text { after PSM }\end{array}$ & No \\
\hline 20. & $\begin{array}{l}\text { Otero-Piñeiro et al. } \\
{[34]}\end{array}$ & $\begin{array}{l}\text { Comparative } \\
\text { cohort study }\end{array}$ & 284 & 100 & $2.5 \%$ vs $11.3 \%$ & Yes \\
\hline 21. & Chang et al. [35] & Cohort study & 110 & 100 & $5.5 \%$ & Not reported \\
\hline
\end{tabular}

$\mathrm{CP}$ - cancer patients, ICG vs. non-ICG - AL rate in ICG vs. non-ICG patients, PSM - propensity score matching

cancer patients. A new evaluation of the AL rate was made, and the difference in AL rate was proved statistically significant, i.e., 3.5\% vs. $10.5 \%$. In their cohort, Santi et al. [22) Indocyanine Green (ICG reported one case of anastomotic leak that occurred on the first postoperative day because of a mechanical problem with the stapler. In a meta-analysis from 2020, the authors reported both a significant reduction in AL rates and a reduction in overall complications when performing ICG angiography [30]. The study by Wada et al. [33] showed an increased anastomotic leak rate when using ICG in the initial data analysis, with a $10.4 \%$ vs. $6.9 \%$ AL rate. However, after propensity score matching, the statistical analysis showed completely opposite results: $8.8 \%$ AL when performing ICG angiography vs.
$14.7 \%$ without angiography.

\section{Study quality and risk of bias}

From the included articles, one was RCT; sixteen were cohort studies, four were meta-analyses or reviews. The studies ${ }^{6}$ levels of evidence according to that of OCEBM were as follows: level one for the meta-analyses, level two for the RCT by De Nardi P, and level three for the cohort studies. The risk of bias was found moderate for all articles, except for the RCT by De Nardi P [16] and the meta-analyses, which were at low risk of bias.

\section{Discussion}

Anastomotic leaks remain a challenge for colorectal surgeons. Even with the use of ERAS 
protocols and many predictive factors, the leaks cannot be wholly evaded as a complication. The consequences of an anastomotic leak are many and various. They are proven to result in increased morbidity, cost of treatment, delay in adjuvant treatment, and mortality. Patients in stage II colorectal cancer with $\mathrm{AL}$ are more than two times more likely to develop a local or distant recurrence [36]. The use of ICG in medicine dates back to 1959 , when it was first approved by the FDA [37]. Recently, using it as a dye for intraoperative immunofluorescent perfusion angiography in colorectal surgery has gained popularity. The method allows for an objective evaluation of the blood supply of the resected colon and rectum and excludes the subjective opinion of the surgeon performing the procedure. The intraoperative assessment of blood supply allows the surgeon to change the transection line or the volume of surgery if insufficient perfusion occurs and may help to reduce the AL rate. However, using ICG angiography does not guarantee $100 \%$ success. Many studies show this by reporting AL rates even after adequate perfusion of the anastomosis is proven. According to the study of Son et al. [18], the positive perfusion test may not be enoughand the fluorescence intensity of colonic flow was measured sequentially, producing perfusion graphs using a video analysis and modeling tool. Colon perfusion patterns were categorized as either fast, moderate, or slow based on their fluorescence slope, T 1/2MAX and time ratio $(\mathrm{TR}=\mathrm{T} 1 / 2 \mathrm{MAX} / \mathrm{T} \mathrm{MAX}$. They suggest a quantitative use of the method, i.e., the presence of coloring from ICG is not enough. The time needed for proving the perfusion test positive also matters. Their results from a logistic regression model showed that slower perfusion is an independent factor for the development of complications in the anastomosis area.

Another interesting result from a metaanalysis published by Zhang et al. [21] showed that using ICG perfusion angiography reduced the leak rate in the patients. However, when changing the transection line, these patients had a higher risk of AL than patients without a resection line change. Therefore, even if we detect an anastomosis at risk of insufficiency because of inadequate blood supply, we are not completely safe from complications developing in the anastomosis area. Another interesting result from the same paper was that seven studies from western countries reported significantly higher ileus rates in the ICG group. In contrast, three studies from East Asia reported significantly lower ileus rates in the ICG group.

The risk of bias was assessed to be moderate for the papers excluding the RCT and metaanalyses, which were at low risk. However, the literature search we performed is also a source of bias. The keywords we used do not guarantee that all studies were included in the review. For example, the FLAG trial [38] and PILLAR II trial [39]mortality, and, in cancer cases, recurrence rates. Inadequate perfusion may contribute to AL. The PINPOINT Endoscopic Fluorescence Imaging System allows for intraoperative assessment of anastomotic perfusion. InStudy Design This is a prospective, multicenter, openlabel, clinical trial that assessed the feasibility and utility of FA for intraoperative perfusion assessment during left-sided colectomy and anterior resection at 11 centers in the United States. $\mid n$ Results A total of 147 patients were enrolled, of whom 139 were eligible for analysis. Diverticulitis (44\% were not returned from the search engine of PUBMED. The subjective selection of keywords by the authors is the major limitation of this review.

Another problem arises from the fact that algorithms for angiography were not reported in all the studies. On the other hand, there were big differences in the reported algorithms. There were neither universal dosages of ICG used nor fixed points at which to perform the angiography. Therefore, efforts should be directed at standardizing the ICG perfusion test.

Even though many of the studies published favor the benefit of ICG angiography, highquality data is missing. The amount of wellplanned and carried out RCT is too scarce. After a number of them are carried out, systematic reviews need to be performed to compare outcomes and provide the data for conclusive answers.

\section{Conclusion}

Anastomotic leak is a severe complication that is a subject of extensive research. Perfusion angiography with ICG is a step towards 
predicting and preventing AL. However, it does not guarantee success in all cases. A standard ICG angiography protocol needs to be developed. More RCTs and well-structured trials on the topic are needed to provide highquality data to be proven safe and beneficial for the patients and be included in the standard therapeutic algorithms.

\section{Acknowledgement}

This article was funded by Medical University Pleven, scientific project №13, and supported by the European Regional Development Fund through the Operational Programme "Science and Education for Smart Growth" under contract №BG05M2OP001-1.0020010-C01(2018-2023).

\section{References}

1. Globocan - The Global Cancer Observatory. Colorectal cancer Source: Globocan 2018. Globocan 2018. 2019;876:1-2.

2. Turrentine FE, Denlinger CE, Simpson VB, Garwood RA, Guerlain S, Agrawal A, et al. Morbidity, mortality, cost, and survival estimates of gastrointestinal anastomotic leaks. J Am Coll Surg. 2015;220(2):195-206.

3. Economic impact of AL in CRC surgery 20052009 USA- SAGES.

4. Nikolian VC, Kamdar NS, Regenbogen SE, Morris AM, Byrn JC, Suwanabol PA, et al. Anastomotic leak after colorectal resection: A population-based study of risk factors and hospital variation. Surg (United States). 2017;161(6):1619-27.

5. Tsujinaka S, Kawamura YJ, Tan KY, Mizokami K, Sasaki J, Maeda T, et al. Proximal bowel necrosis after high ligation of the inferior mesenteric artery in colorectal surgery. Scand J Surg. 2012;101(1):21-5.

6. Stewart LA, Clarke M, Rovers M, Riley RD, Simmonds M, Stewart G, et al. Preferred reporting items for a systematic review and meta-analysis of individual participant data: The PRISMA-IPD statement. JAMA - J Am Med Assoc. 2015;313(16):1657-65.

7. OCEBM Levels of Evidence Working G. "The Oxford Levels of Evidence 2" 2011

8. Sterne JA, Hernán MA, Reeves BC, Savović J, Berkman ND, Viswanathan $M$, et al. ROBINS-I: A tool for assessing risk of bias in non-randomised studies of interventions. BMJ.
2016;355:4-10.

9. Koh FH, Tan KK. Fluorescent Angiography Used to Evaluate the Perfusion Status of Anastomosis in Laparoscopic Anterior Resection. Ann Surg Oncol. 2016;23(August):692.

10. Keller DS, Boulton R, Rodriguez-Justo M, Cohen R, Chand M. A Novel Application of Indocyanine Green Immunofluorescence in Emergent Colorectal Surgery. J Fluoresc. 2018;28(2):487-90.

11. Ryu S, Yoshida M, Hironori O, Tsutsui N, Suzuki $\mathrm{N}$, Ito $\mathrm{E}$, et al. Intraoperative ICG fluorescence contrast imaging of the main artery watershed area in colorectal cancer surgery: Report of a case. Int J Surg Case Rep. 2016;26:176-8.

12. Shigoka M, Hidaka E, Tomita K, Nakagawa M, Yokozuka K, Miyoshi K, et al. A Case of Left Transverse Colon Cancer Safely Resected Using Three-Dimensional CT Angiography and Indocyanine Green Fluorography. Gan To Kagaku Ryoho. 2020 Dec;47(13):2308-10.

13. Maehara Y, Soejima Y, Yoshizumi T, Kawahara $\mathrm{N}$, Oki E, Saeki H, et al. The evolution of surgical treatment for gastrointestinal cancers. Int J Clin Oncol. 2019;24(11):1333-49.

14. 14. D’Urso A, Agnus V, Barberio M, Seeliger B, Marchegiani F, Charles AL, et al. Computerassisted quantification and visualization of bowel perfusion using fluorescence-based enhanced reality in left-sided colonic resections. Surgical Endoscopy. 2020.

15. Brescia A, Pezzatini M, Romeo G, Cinquepalmi M, Pindozzi F, Dall'Oglio A, et al. Indocyanine green fluorescence angiography: a new ERAS item. Updates Surg. 2018;70(4):427-32.

16. De Nardi P, Elmore U, Maggi G, Maggiore $\mathrm{R}$, Boni L, Cassinotti $\mathrm{E}$, et al. Intraoperative angiography with indocyanine green to assess anastomosis perfusion in patients undergoing laparoscopic colorectal resection: results of a multicenter randomized controlled trial. Surg Endosc. 2020;34(1):53-60.

17. Hasegawa H, Tsukada Y, Wakabayashi M, Nomura S, Sasaki T, Nishizawa Y, et al. Impact of intraoperative indocyanine green fluorescence angiography on anastomotic leakage after laparoscopic sphincter-sparing surgery for malignant rectal tumors. Int $\mathrm{J}$ Colorectal Dis. 2020;35(3):471-80.

18. Son GM, Kwon MS, Kim Y, Kim J, Kim $\mathrm{SH}$, Lee JW. Quantitative analysis of colon perfusion pattern using indocyanine green (ICG) angiography in laparoscopic colorectal surgery. Surg Endosc. 2019;33(5):1640-9.

19. Boni L, Fingerhut A, Marzorati A, Rausei S, Dionigi G, Cassinotti E. Indocyanine green fluorescence angiography during laparoscopic 
low anterior resection: results of a case-matched study. Surg Endosc. 2017;31(4):1836-40.

20. Ishii M, Hamabe A, Okita $K$, Nishidate $T$, Okuya K, Usui A, et al. Efficacy of indocyanine green fluorescence angiography in preventing anastomotic leakage after laparoscopic colorectal cancer surgery. Int J Colorectal Dis. 2020;35(2):269-75.

21. Zhang W, Che X. Effect of indocyanine green fluorescence angiography on preventing anastomotic leakage after colorectal surgery: a meta-analysis. Surg Today. 2021 Jan 11.

22. Santi C, Casali L, Franzini C, Rollo A, Violi V. Applications of indocyanine green-enhanced fluorescence in laparoscopic colorectal resections. Updates in Surgery. 2019;71:83-8.

23. Liu D, Liang L, Liu L, Zhu Z. Does intraoperative indocyanine green fluorescence angiography decrease the incidence of anastomotic leakage in colorectal surgery? A systematic review and meta-analysis. Int $\mathrm{J}$ Colorectal Dis. 2021;36(1):57-66.

24. Lin J, Zheng B, Lin S, Chen Z, Chen S. The efficacy of intraoperative ICG fluorescence angiography on anastomotic leak after resection for colorectal cancer: a meta-analysis. Int $\mathrm{J}$ Colorectal Dis. 2021 Jan 4;36(1):27-39.

25. Boni L, David G, Dionigi G, Rausei S, Cassinotti $\mathrm{E}$, Fingerhut A. Indocyanine green-enhanced fluorescence to assess bowel perfusion during laparoscopic colorectal resection. Surg Endosc. 2016;30(7):2736-42.

26. Bonadio L, Iacuzzo C, Cosola D, Cipolat Mis T, Giudici F, Casagranda B, et al. Indocyanine green-enhanced fluorangiography (ICGf) in laparoscopic extraperitoneal rectal cancer resection. Updates in Surgery. 2020;72:477-82.

27. Benčurik V, Škrovina M, Martínek L, Bartoš J, Macháčková M, Dosoudil M, et al. Intraoperative fluorescence angiography and risk factors of anastomotic leakage in mini-invasive low rectal resections. Surg Endosc. 2020;(0123456789).

28. Carus T, Pick P. Intraoperative fluorescence angiography in colorectal surgery. Chirurg. 2019;90(11):887-90.

29. Protyniak B, Dinallo AM, Boyan WP, Dressner RM, Arvanitis ML. Intraoperative indocyanine green fluorescence angiography - An objective evaluation of anastomotic perfusion in colorectal surgery. Am Surg. 2015;81(6):580-4.

30. Li Z, Zhou Y, Tian G, Liu Y, Jiang Y, Li $\mathrm{X}$, et al. Meta-Analysis on the Efficacy of Indocyanine Green Fluorescence Angiography for Reduction of Anastomotic Leakage After Rectal Cancer Surgery. Am Surg. 2020 Dec 30;000313482098284.
31. Ris F, Hompes R, Cunningham C, Lindsey I, Guy $\mathrm{R}$, Jones $\mathrm{O}$, et al. Near-infrared (NIR) perfusion angiography in minimally invasive colorectal surgery. Surg Endosc. 2014;28(7):2221-6.

32. Gröne J, Koch D, Kreis ME. Impact of intraoperative microperfusion assessment with Pinpoint Perfusion Imaging on surgical management of laparoscopic low rectal and anorectal anastomoses. Color Dis. 2015;17:228.

33. Wada T, Kawada K, Hoshino N, Inamoto S, Yoshitomi M, Hida K, et al. The effects of intraoperative ICG fluorescence angiography in laparoscopic low anterior resection: a propensity score-matched study. Int $\mathrm{J}$ Clin Oncol. 2019;24(4):394-402.

34. Otero-Piñeiro AM, de Lacy FB, Van Laarhoven JJ, Martín-Perez B, Valverde S, Bravo R, et al. The impact of fluorescence angiography on anastomotic leak rate following transanal total mesorectal excision for rectal cancer: a comparative study. Surg Endosc. 2021;35(2):754-62.

35. Chang YK, Foo CC, Yip J, Wei R, Ng KK, Lo $\mathrm{O}$, et al. The impact of indocyanine-green fluorescence angiogram on colorectal resection. Surgeon. 2019;17(5):270-6.

36. Fang C, Nie P, Jing P, Zhang Y, Yang L, Yu Y, et al. Effects of Adjuvant Therapy Compliance and Anastomotic Leakage on the Oncologic Outcomes of Patients With Rectal Cancer After Curative Resection. Dis Colon Rectum. 2020 Dec 17.

37. Mizrahi I, Abu-Gazala M, Rickles AS, Fernandez LM, Petrucci A, Wolf J, et al. Indocyanine green fluorescence angiography during low anterior resection for low rectal cancer: results of a comparative cohort study. Tech Coloproctol. 2018;22(7):535-40.

38. Alekseev M, RybakovE, Shelygin Y, Chernyshov S, Zarodnyuk I. A study investigating the perfusion of colorectal anastomoses using fluorescence angiography: results of the FLAG randomized trial. Color Dis. 2020;22(9):114753.

39. Jafari MD, Wexner SD, Martz JE, McLemore EC, Margolin DA, Sherwinter DA, et al. Perfusion assessment in laparoscopic left-sided/anterior resection (PILLAR II): A multi-institutional study. J Am Coll Surg. 2015;220(1):82-92.e1. 